\title{
Dopaminergic neuronal development in the embryonic mesencephalon of mouse
}

\author{
Mun-Ki Kim, Si-Joon Lee, Chung-Kil Won* \\ Institute of Animal Medicine and Department of Veterinary Medicine, Gyeongsang National University, Jinju 52828, Korea
}

\begin{abstract}
This study presents neuronal migration pattern of dopamine (DA) neurons generated in separate regions occupying the ventral mesencephalic territory. A single pulse 5-bromodeoxyuridine (BrdU) was administered at embryonic day (E)10-E15. Distribution of tyrosine hydroxylase (TH) positive cells was determined at E13-postnatal day 0 (P0) by immunohistochemistry. BrdU positive cells labeled at E10 were spread out uniformly in the mesencephalon from E13 to E15, migrating through dorsal and ventral routes at E17 and P0. TH expression labeled at E10 was observed at E13 in the ventromedial region and clearly formed in the ventral tegmental area (VTA) at E15. At E17, TH expression in the substantia nigra (SN) was observed in the ventrolateral region, spreading more outward of the mesencephalon at P0. Generation of TH-positive cells labeled at E13 was also observed in VTA and SN of the mesencephalon at E17 and P0. The expression of these cells labeled after E15 was markedly decreased. These results demonstrated that an almost complete primary structure of DA neuron was formed at the early embryonic stage in the ventral mesencephalon, showing the most active neuronal migration was occurred at E13-E17.
\end{abstract}

Keywords: development, dopaminergic neuron, mesencephalon, neuronal migration

*Corresponding author

Chung-Kil Won

Institute of Animal Medicine and Department of Veterinary Medicine, Gyeongsang National University, 501 Jinju-daero, Jinju 52828, Korea

Tel: $+82-55-772-2351$

Fax: +82-55-772-2349

E-mail:wonck@gnu.ac.kr

ORCID:

Chung-Kil Won

https://orcid.org/0000-0002-3105-1869

Mun-Ki Kim

https://orcid.org/0000-0002-7397-2236

Si-Joon Lee

https://orcid.org/0000-0002-5870-687X

Conflict of Interest

The authors declare no conflicts of interest.

Received: September 18, 2020

Revised: October 19, 2020

Accepted: October 25, 2020

\section{Introduction}

Neuronal and non-neuronal cells are usually generated at different areas where they eventually reside. Neurons originate from the site of their last mitotic division such as the ventricular zone. They then migrate to their final destination and become integrated into specific brain circuits [1-5]. Neuronal migration is an important development process in the central nervous system [2-7]. However, neuronal migration remains largely unexplored in mouse mesencephalon where dopamine (DA) neurons occupy a major part in ventral mesencephalon [8-9].

DA neurons in the mesencephalon are distributed into three major cell groups: ventral tegmental area (VTA), substantia nigra (SN), and retrorubral field (RRF) [10]. Mesencephalic SN cells project to the dorsolateral striatum, establishing the nigrostriatal pathway. VTA and RRF cells innervate to the ventromedial striatum, amygdala, olfactory tubercle, thalamus, and hippocampus, forming the mesolimbic pathway [11]. DA neurons in the midbrain can modulate cognitive function, regulate voluntary movement, and encode reward prediction errors and aversive stimuli. While degeneration of DA neuros underlies motor defects in Parkinson's disease, imbalances in DA levels are associated with neuropsychiatric disorders such as depression, schizophrenia, and substance abuse $[12,13]$.

During development, postmitotic DA neurons are known to migrate away from the ventricular surface toward the ventromedial mesencephalon along radial glial fibers. They then migrate laterally along tangentially arranged nerve fibers $[14,15]$. However, the neuronal migration pattern of DA neurons in the developmental stage of mesencephalon is not well understood yet. Thus, the objective of this study was to characterize neuronal migration of DA neurons in early and late embryonic mesencephalon of mouse.

\section{Materials and Methods}

\section{Animals}

Timed pregnant CD1 mice were purchased from Charles River Laborato- 
ries (USA) and maintained in an animal facility. The day of plug discovery was designated as embryonic day 0 (E0). Embryos were removed after deep inhalation anesthesia by the mother of mouse with isoflurane. Three mice were used for each group regardless of sex (male or female). Animal experiments were in full compliance with the NIH Guide for the Care and Use of Laboratory Animals and approved by McLean Institutional Animal Care and Use Committee.

5-Bromodeoxyuridine labeling and immunohistochemistry For neuronal migration study, a single 5-bromodeoxyuridine (BrdU: $50 \mu \mathrm{g} / \mathrm{g}$ body weight, i.p; Sigma) injection was administrated to E10, E11, E13, and E15 pregnant dams.
Embryos were removed at E13, E15, E17, and postnatal day 0 (P0) and decapitated. Embryonic brains were immersed in Zinc fixative (BD Pharmingen, USA) for 24 hours and then processed for paraffin wax histology. BrdU immunohistochemistry was performed for $10 \mu \mathrm{m}$ thick paraffin embedded sections with a mouse monoclonal anti-BrdU antibody (1:75, 347580, BD Pharmingen). Double labeling immunohistochemistry for BrdU and tyrosine hydroxylase (TH) was performed using rabbit TH antibody (1:1000, P40101-0, PelFreez, USA). Streptavidin Alexa fluor 488 conjugated antirabbit IgG (1:400, Molecular Probes, USA), and Streptavidin Alexa fluor 594 conjugated anti-mouse $\operatorname{IgG}$ (1:400, Molecular Probes) were used as secondary antibodies.
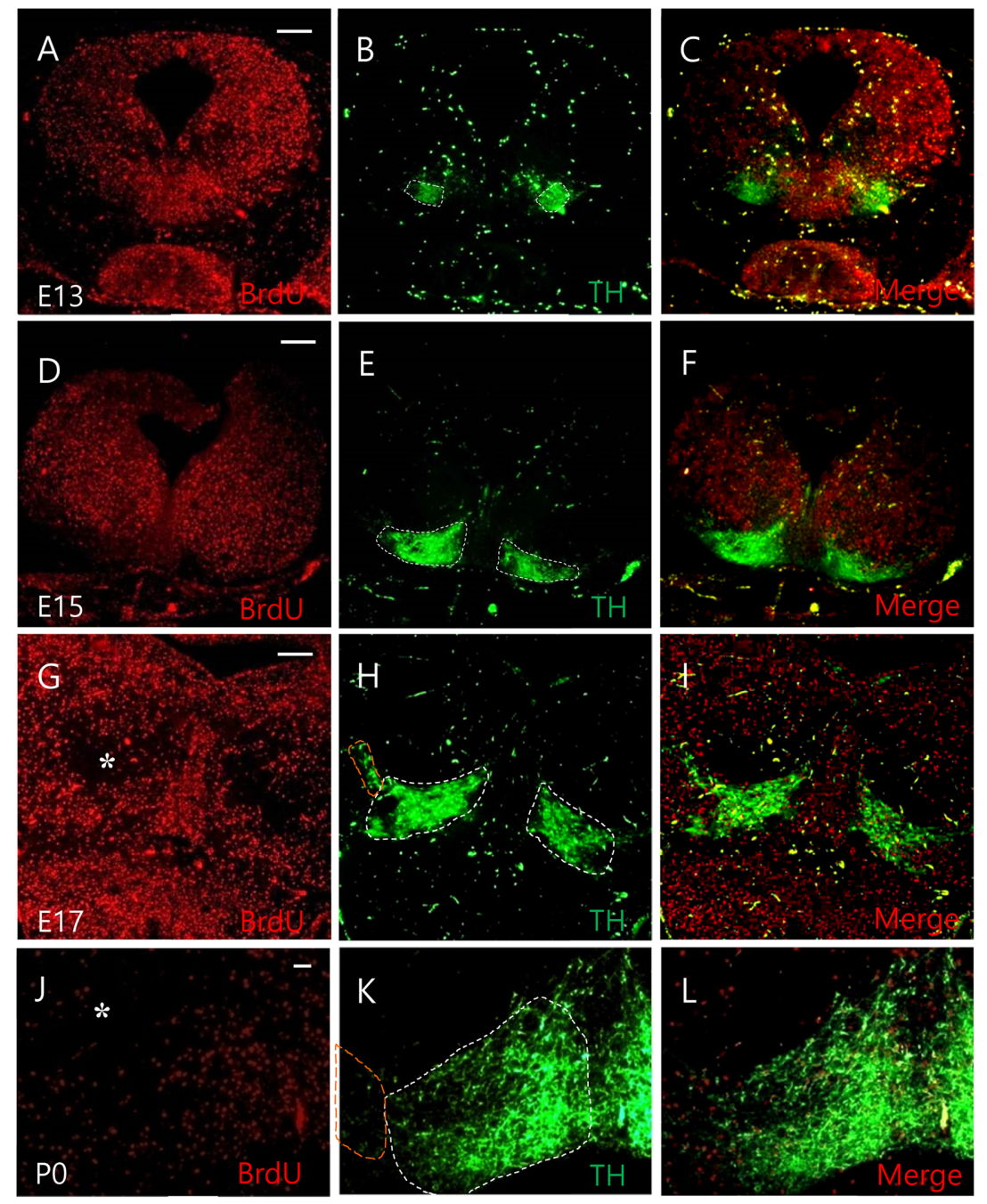

Fig. 1. Neuronal migration of dopamine neurons labeled with BrdU at E10. TH expression in embryonic development of the mesencephalon was determined. BrdU was administrated at E10. TH positive cells at E13, E15, E17, and P0 were then determined. BrdU positive cells were spread out uniformly at E13, E15 (A, D) and migrated through dorsal and ventral routes at E17 and P0 (G, J). TH expression was observed in the ventromedial region. TH/BrdU double labeled cells were identified at E13 (B, C). TH expression at E15 formed a clear structure in the VTA (E, F). At E17, TH expression was observed in the VTA and SN (H, I) and spread further outward of the mesencephalon at P0 (K, L). At P0, BrdU cells merged with TH positive cell in the VTA and SN. White dashed line represents VTA, yellow dashed line represents SN. Scale bars: $100 \mu \mathrm{m}$ in A-I, $10 \mu \mathrm{m}$ in J-L. VTA, ventral tegmental area; TH, tyrosine hydroxylase; $\mathrm{SN}$, substantia nigra. 


\section{Results}

BrdU birthdating studies have been widely used to study neuronal migration in the developing neocortex [16-18]. Because BrdU is integrated into the DNA of S-phase progenitor cells, it serves as a stable marker for cells born around the time of injection. We performed a thorough and systematic BrdU birthdating study to understand mesencephalic neuronal migration in CD1 mice. In this study, no change in the number of offspring or brain tissue was observed following administration of BrdU. To investigate neuronal migration of DA neurons in embryonic development of the mesencephalon, a single pulse BrdU was administrated at E10. Distribution of TH positive cells was then determined at E13, E15, E17, and P0, respectively. BrdU positive cells labeled at E10 were spread out uniformly in the mesencephalon at E13 and E15 (Fig. 1A and D), migrating through dorsal and ventral routes at E17 and P0 (Fig. 1G and J). These changed distribution patterns of BrdU positive cells labeled at E10 were more clearly observed at P0, but were rarely observed in the entire mesencephalon.

TH expression labeled at E10 was observed in the ventromedial region of the mesencephalon. TH/BrdU double labeled cells were identified at E13 (Fig. 1B and C). At E15, $\mathrm{TH}$ expression was formed in an anatomically clear structure in the VTA of the midbrain (Fig. 1E and F). At E17, TH expression was observed not only in the VTA region, but also in the $\mathrm{SN}$ of the ventrolateral region (Fig. $1 \mathrm{H}$ and I), spreading more outward of the mesencephalon at P0 (Fig. 1K and L). Some BrdU positive cells labeled at E10 merged with TH positive cell at E17 and P0 in the VTA and SN of the mesencephalon (Fig. 1I and L).

To investigate when and where DA neurons were migrated in the mesencephalon, BrdU was injected into CD1 mice at E13 and E15. Each brain sample was obtained at E17 and $\mathrm{P} 0$, respectively. BrdU positive cells labeled at E13 were observed to migrate out through dorsal and ventral routes at E17 (Fig. 2A). These changed distribution patterns of cells labeled at E13 were clearly observed at P0 (Fig. 3A). BrdU positive cells labeled at E15 were rarely observed in the
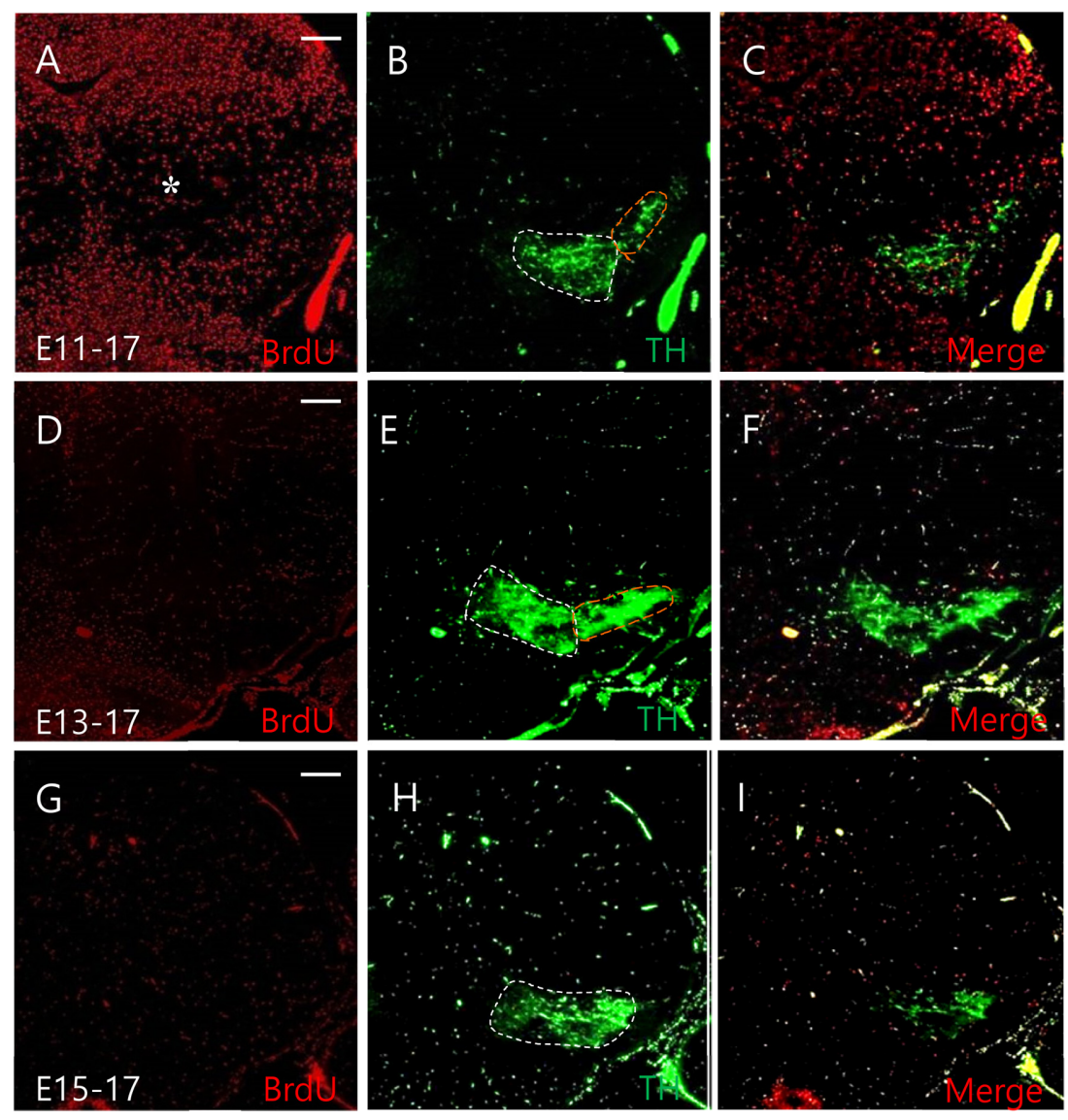

Fig. 2. Neuronal migration of dopamine neurons observed at E17. BrdU was injected at E13 and E15. Each brain sample was obtained at E17. BrdU positive cells labeled at E13 were observed to migrate out through dorsal and ventral routes at E17 (A). BrdU cells labeled at E15 were rarely observed in the entire mesencephalon at E17 (D). TH positive cells labeled at E13 were also found in the VTA and SN at E17 (B, C). However, the expression of these cells labeled at E15 was reduced at P0 (E, F). White dashed line represents VTA, yellow dashed line represents SN. Scale bars: $100 \mu \mathrm{m}$ in A-F. VTA, ventral tegmental area; TH, tyrosine hydroxylase; $\mathrm{SN}$, substantia nigra. 

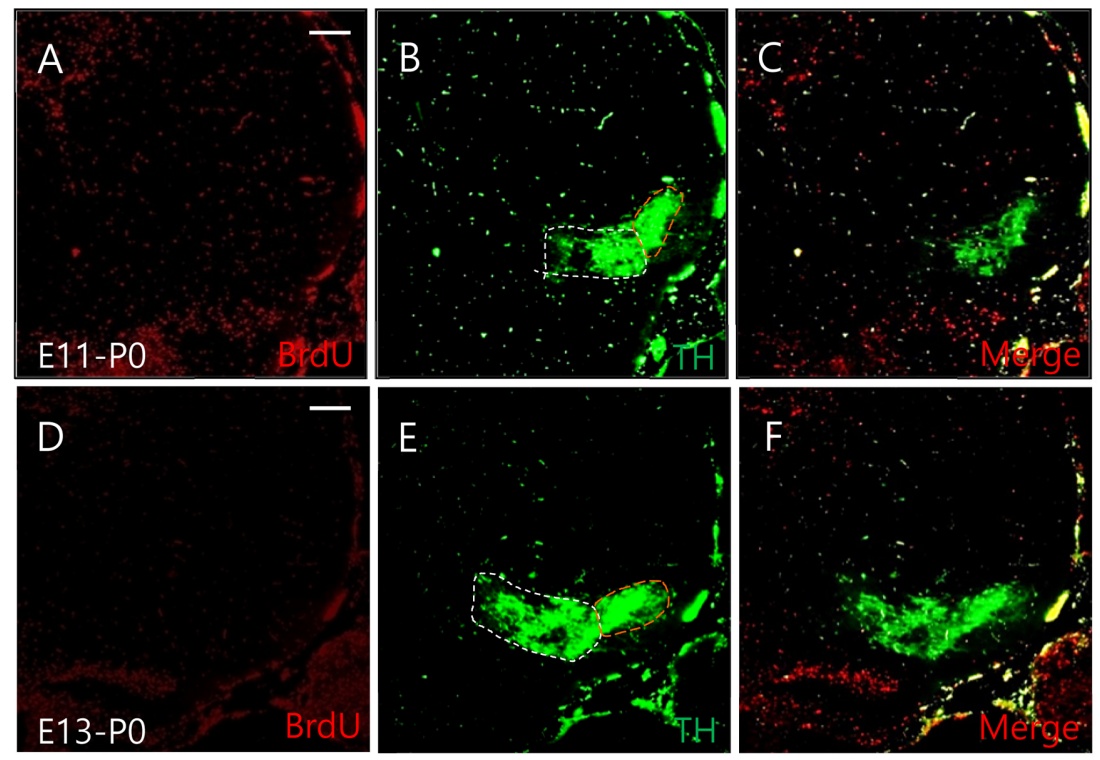

Fig. 3. Neuronal migration of dopamine neurons observed at P0. BrdU was injected at E13 and E15. Each brain sample was obtained at P0. BrdU positive cells labeled at E13 and E15 were clearly observed in the entire mesencephalon at P0 (A, D). At P0, the expression of TH-positive cells labeled at E13 was observed (B, C). However, cells labeled at E15 were reduced compared to cells expressed at E13-P0 (E, F). White dashed line represents ventral tegmental area, yellow dashed line represents substantia nigra. Scale bars: $100 \mu \mathrm{m}$ in A-F. TH, tyrosine hydroxylase.

entire mesencephalon at E17 and P0 (Figs. 2D and 3D).

We then double-labeled cells with TH/BrdU at E17 and P0 to determine when TH-positive cells labeled at E13 and E15 would migrate during the development of the mesencephalon. The expression of TH positive cells labeled at E13 was also found in the VTA and SN of the mesencephalon at E17 (Fig. 2B and C). However, the expression of these cells labeled at E15 was reduced at P0 (Fig. 2E and F). At P0, the expression of $\mathrm{TH}$-positive cells labeled at E13 was higher than that at E13-E17 (Fig. 3B and C). These cells labeled at E15 were reduced than those expressed at E13-P0 (Fig. 3E and F). In this experiment, different expression was observed between cells labeled from E10 to E15, with cells labeled between E13-E17 showing strong TH neuronal migration patterns in the VTA and SN of the mesencephalon.

\section{Discussion}

Neuronal migration is an essential step in the formation of the anatomical structure of the mesencephalon. One important feature of neurons in the developing central nervous system is that they can migrate from the embryonic region of origin to their final destination [2]. Cells generated in the ventral mesencephalon can migrate ventrally and dorsally [18]. In this study, BrdU immunohistochemistry showed evidence of neuronal migration in the mesencephalon. E10labeled BrdU positive cells were uniformly distributed at E13 to E15. However, these cells became diminished in the red nucleus area at E17. This depletion of cells is considered to be a consequence of dorsal and ventral neuronal migration
[19]. Although a minor difference was observed between the migration of E10-labeled cells, they showed the same dorsal and ventral migration pattern. TH mRNA was detected at E11.5 by in situ hybridization. It was first detected at E11.5 by real time reverse transcription-polymerase chain reaction [20]. In addition, developmental studies of DA neurons have shown that neurogenesis begins at E10-E11 in mouse mesencephalon. After E12, most of BrdU-positive cells are expressed in the dorsal region of the midbrain, whereas TH-positive cells are expressed only in the dorsal region of the mesencephalon [19]. However, most reports on the development of DA neurons are about early neurogenesis. There are few reports on the migration of DA neurons during neurogenesis stage in the mesencephalon.

The migratory mode of DA neurons is divided into two phases [14,15,21]. First, early mesencephalic DA neurons from the ventricular surface will migrate to the ventromedial mesencephalon along radial glial fibers. Second, they will migrate laterally in the basal part of ventral mesencephalon along tangentially arranged nerve fibers to form anatomically specified cell groups [18]. The present study demonstrated that DA neurons were initially generated in the ventromedial region of the mesencephalon. After migrating to the VTA, they then migrated to the SN in the mesencephalon.

In the early embryonic state of E10-E12, DA neurogenesis was observed throughout the mesencephalon. This DA neurogenesis has only been confirmed in ventromedial regions after E12 [19]. We induced TH/BrdU double expression in $\mathrm{CD} 1$ mice to investigate the migration of DA neurons during the development of mesencephalons in the embryonic stage. 
At E10-E13, the early embryonic stage, DA neurons migrated to form part of the ventromedial region of mesencephalon. At E15, DA neurons were observed to form a well-defined VTA in the ventral region of the mesencephalon. Our results also confirmed that DA neurons migrated to SN of mesencephalon after E17. These results demonstrate that DA neurons in VTA mainly consist of neurons generated at the beginning of the development and that DA neurons in SN mainly migrate after E17. On the other hand, in SN, DA neurons migrating from E13 showed a similar pattern to cells migrating from E10. These results suggest that DA neurons of mesencephalic SN mainly migrate DA neurons after E13.

On the other hand, GABAergic and dopaminergic neurons in the mesencephalon are predominantly originated at E10 to E11 [18,19]. Major neurogenesis of GABAergic neurons occurs at E10 to E13. Neuronal migration continues until neonatal period during mesencephalic development [22]. The present study revealed that DA neurons were initially detected at E13 and that many E10-labeled BrdU positive cells merged with DA neurons in the SN and VTA at P0. These results are similar to the migration of GABA neurons.

In conclusion, expression of TH-positive cells was labeled at E10-E15, and the results of investigation at 17 and P0 revealed that DA neurons generated in the VTA and SN regions of mesencephalon were the most active neuron migration in E13-E17.

\section{Acknowledgments}

This research was supported by Basic Science Research Program through the National Research Foundation of Korea (2018R1D1A1B0704925813) funded by the Ministry of Education.

\section{References}

1. Corbin JG, Nery S, Fishell G. Telencephalic cells take a tangent: non-radial migration in the mammalian forebrain. Nat Neurosci 2001;4 Suppl:1177-1182.

2. Hatten ME. New directions in neuronal migration. Science 2002;297:1660-1663.

3. Marín O, Rubenstein JL. A long, remarkable journey: tangential migration in the telencephalon. Nat Rev Neurosci 2001;2: 780-790.

4. Nadarajah B, Parnavelas JG. Modes of neuronal migration in the developing cerebral cortex. Nat Rev Neurosci 2002;3: 423-432.

5. Parnavelas JG. The origin and migration of cortical neurones: new vistas. Trends Neurosci 2000;23:126-131.

6. Altman J, Bayer SA. Development of the brain stem in the rat. V. Thymidine-radiographic study of the time of origin of neurons in the midbrain tegmentum. J Comp Neurol 1981; 198:677-716.
7. Smits SM, Burbach JP, Smidt MP. Developmental origin and fate of meso-diencephalic dopamine neurons. Prog Neurobiol 2006;78:1-16.

8. Korotkova TM, Ponomarenko AA, Brown RE, Haas HL. Functional diversity of ventral midbrain dopamine and GABAergic neurons. Mol Neurobiol 2004;29:243-259.

9. Nair-Roberts RG, Chatelain-Badie SD, Benson E, WhiteCooper H, Bolam JP, Ungless MA. Stereological estimates of dopaminergic, GABAergic and glutamatergic neurons in the ventral tegmental area, substantia nigra and retrorubral field in the rat. Neuroscience 2008;152:1024-1031.

10. German DC, Manaye KF. Midbrain dopaminergic neurons (nuclei A8, A9, and A10): three-dimensional reconstruction in the rat. J Comp Neurol 1993;331:297-309.

11. Prakash N, Wurst W. Development of dopaminergic neurons in the mammalian brain. Cell Mol Life Sci 2006;63:187-206.

12. Bodea GO, Blaess S. Establishing diversity in the dopaminergic system. FEBS Lett 2015;589 24 Pt A:3773-3785.

13. Damier P, Hirsch EC, Agid Y, Graybiel AM. The substantia nigra of the human brain. II. Patterns of loss of dopaminecontaining neurons in Parkinson's disease. Brain 1999;122: 1437-1448.

14. Kawano H, Ohyama K, Kawamura K, Nagatsu I. Migration of dopaminergic neurons in the embryonic mesencephalon of mice. Brain Res Dev Brain Res 1995;86:101-113.

15. Ohyama K, Kawano H, Asou H, Fukuda T, Oohira A, Uyemura K, Kawamura K. Coordinate expression of L1 and $6 \mathrm{~B} 4$ proteoglycan/phosphacan is correlated with the migration of mesencephalic dopaminergic neurons in mice. Brain Res Dev Brain Res 1998;107:219-226.

16. López-Bendito G, Sánchez-Alcañiz JA, Pla R, Borrell V, Pic E, Valdeolmillos M, Marín O. Chemokine signaling controls intracortical migration and final distribution of GABAergic interneurons. J Neurosci 2008;28:1613-1624.

17. Soriano E, Del Rio JA. Simultaneous immunocytochemical visualization of bromodeoxyuridine and neural tissue antigens. J Histochem Cytochem 1991;39:255-263.

18. Vasudevan A, Won C, Li S, Erdélyi F, Szab G, Kim KS. Dopaminergic neurons modulate GABA neuron migration in the embryonic midbrain. Development 2012;139:3136-3141.

19. Kim MK, Lee SJ, Vasudevan A, Won C. Neurogenesis and neuronal migration of dopaminergic neurons during mesencephalon development in mice. J Biomed Transl Res 2018; 19:125-129.

20. Castelo-Branco G, Wagner J, Rodriguez FJ, Kele J, Sousa K, Rawal N, Pasolli HA, Fuchs E, Kitajewski J, Arenas E. Differential regulation of midbrain dopaminergic neuron development by Wnt-1, Wnt-3a, and Wnt-5a. Proc Natl Acad Sci U S A 2003;100:12747-12752.

21. Shults CW, Hashimoto R, Brady RM, Gage FH. Dopaminergic cells align along radial glia in the developing mesencephalon of the rat. Neuroscience 1990;38:427-436.

22. Kim MK, Lee SJ, Vasudevan A, Won CK. GABAergic neuronal development in the embryonic mesencephalon of mice. Korean J Vet Res 2019;59:201-205. 